\title{
Presence of Non-dynamical Fluctuations in the Higher Moments of Net-proton Multiplicity Distribution
}

\section{Prakhar Garg*}

Department of Physics and Astronomy, Stony Brook University, Stony Brook 11794-3800, USA

E-mail: prakhar.gargestonybrook. edu

\section{Dipak Kumar Mishra}

Nuclear Physics Division, Bhabha Atomic Research Center, Mumbai 400085, India

E-mail: dkmishra@rcf.rhic.bnl.gov

\begin{abstract}
Recent results on net-proton multiplicity fluctuations of the STAR experiment from the beam energy scan (BES) program at the BNL Relativistic Heavy Ion Collider (RHIC) have drawn much attention to explore the QCD critical point and the nature of the deconfinement phase transition. The non-monotonic behavior of $\kappa \sigma^{2}$ around $\sqrt{s_{N N}}=19.6 \mathrm{GeV}$ in the STAR results even increased the excitement in the field, as it may be associated with the existence of a QCD critical point. Although the presence of many statistical fluctuations become the background for the dynamical fluctuations and it is important to disentangle and identify them to have a correct interpretation. The recent findings for the stopped proton fluctuations and event-pile up scenarios as a background and their influence on the interpretation of net-proton fluctuations are discussed in the present work.
\end{abstract}

Critical Point and Onset of Deconfinement - CPOD2017

7-11 August, 2017

The Wang Center, Stony Brook University, Stony Brook, NY

\footnotetext{
*Speaker.
} 


\section{Introduction}

Exploration of the phase diagram of strong interaction is one of the main motivation of Beam Energy Scan (BES) program at RHIC besides other heavy-ion experiments and upcoming facilities. Quantum Chromodynamics (QCD) calculations by lattice indicates a smooth crossover at finite baryon chemical potential $\left(\mu_{B}\right) \approx 0$ ) while other models predict a first order phase transition at higher $\mu_{B}$ in the QCD phase diagram. The coexistence of crossover and first order phase transition in the QCD phase diagram suggests an existence of the QCD critical end point (CEP) [1,2].

The event-by-event fluctuations of conserved quantities for example net-baryon number, netcharge, and net-strangeness are proposed as a signatures of the existence of CEP as they are sensitive to the correlation length. Since the correlation length $(\xi)$ of the system is related to the moments of the multiplicity distribution of these conserved quantities, which can be used to look for phase transition and CEP by varying the center of mass energy of the colliding nuclei. The variance $\left(\sigma^{2}\right)$, skewness $(S)$ and kurtosis $(\kappa)$ of these distributions are related to $\xi$ as $\xi^{2}, \xi^{4.5}$ and $\xi^{7}$, respectively [2]. Since the higher order moments have more sensitivity to the correlation length, studying them for the conserved number distributions can amplify the signal arises due to dynamical fluctuation.

The shape of the net-distribution is described by the moments (mean, $\sigma, S$, and $\kappa$ ) and they are related to the cumulants $\left(C_{n}, n=1,2,3,4\right)$ as: mean $(M)=C_{1}, \sigma^{2}=C_{2}=\left\langle(\Delta N)^{2}\right\rangle, S=C_{3} / C_{2}^{3 / 2}=$ $\left\langle(\Delta N)^{3}\right\rangle / \sigma^{3}$ and $\kappa=C_{4} / C_{2}^{2}=\left\langle(\Delta N)^{4}\right\rangle / \sigma^{4}-3$, where $N$ is the multiplicity of the net distributions and $\Delta N=N-M$. Further, the ratio of various $n^{\text {th }}$ order cumulants $C_{n}$ of the distributions are related to the ratios and product of the moments as: $\sigma^{2} / M=C_{2} / C_{1}, S \sigma=C_{3} / C_{2}, \kappa \sigma^{2}=C_{4} / C_{2}$, and $S \sigma^{3} / M=C_{3} / C_{1}$. Also, the cumulant ratios can be related to the generalized susceptibilities calculated in lattice QCD and other statistical models [3, 4].

The measurements of net-proton and net-charge multiplicity distributions from BES at RHIC have drawn much attention from both the theoretical and the experimental community $[5,6]$. There have been speculations that, the non-monotonic behavior of $\kappa \sigma^{2}$ as a function of center of mass energies $\left(\sqrt{s_{N N}}\right)$ in STAR net-proton results may be an indication of the QCD critical point. Several studies have been performed afterwards to investigate the excess of dynamical fluctuations such as the effect of kinematical acceptance, inclusion of resonance decays, exact (local) charge conservation, excluded volume corrections and so forth for a proper thermal baseline to experimental measurements in order to draw physics conclusions. However, in this presentation mainly two points are discussed; Firstly a possibility of event pile-up as an artifact which can be present in these measurements and the effect of stopped proton fluctuations on these observables.

We demonstrate that how a small fraction of residual pile-up events can change the moments and their ratios of an event-by-event net-proton multiplicity distribution. We emphasize that, it might be crucial to estimate the fraction of pile-up events in the data sample while interpreting the experimental observable for the critical point. Further, the experimentally measured protons contain contribution from higher mass resonance decays, production process, and protons from the baryon stopping [10] and these contributions fluctuate from event to event, which can contaminate the dynamical fluctuations. In the present work, we also estimate the contribution of stopped proton and produced proton in the net-proton multiplicity fluctuations in $\mathrm{Au}+\mathrm{Au}$ collisions measured by STAR experiment at RHIC at different collision energies. 


\section{Contribution of Event Pile-up Background}

High-luminosity modern accelerators, like the Relativistic Heavy Ion Collider (RHIC) at Brookhaven National Laboratory (BNL) and Large Hadron Collider (LHC) at European Organization for Nuclear Research (CERN), inherently have event pile-up scenarios which significantly contribute to physics events as a background. While the tracking algorithms and detector concepts take care of most of the event pile-up scenarios, several offline analytical techniques are also used to remove such events for the physics analysis. It is still difficult to identify the remaining pile-up events in an event sample for physics analysis. Since the fraction of these events is significantly small, it may not be as serious of an issue for other analyses as it would be for an event-by-event analysis. In the present work, we demonstrate how a small fraction of residual pile-up events can change the moments and their ratios of an event-by-event net-proton multiplicity distribution, which are sensitive to the dynamical fluctuations due to the QCD critical point [7].

In case of high luminosity accelerators, the contributions to background events may include from the In-time pile-up, Out-of-time pile-up, Cavern background, Beam halo and Beam gas events. As an example, the average store luminosity of $1.3 \times 10^{26} \mathrm{~cm}^{-2} \mathrm{sec}^{-1}$ and the collision cross-section of 9.6 barn has been measured by the STAR experiment for $19.6 \mathrm{GeV} \mathrm{Au}+\mathrm{Au}$ Run. Therefore, the collision rate was around $1.25 \times 10^{3} \mathrm{sec}^{-1}(\approx 1.25 \mathrm{kHz})$. Further, the time difference between two bunches at RHIC is $109 \mathrm{~ns}$, hence the contribution to the in-time pile-up will be $109 \mathrm{~ns}$ $\times 1.25 \mathrm{kHz}=1.36 \times 10^{-4}$ at $\sqrt{s_{N N}}=19.6 \mathrm{GeV}$. Similarly, the collision rates for $\mathrm{Au}+\mathrm{Au}$ collisions at $\sqrt{s_{N N}}=200 \mathrm{GeV}$ are around $60 \mathrm{kHz}$, which leads to $\sim 6.5 \times 10^{-3}$ events as in-time pileup.

For this study, we assume that the individual event-by-event proton and antiproton multiplicity distributions follow binomial distributions, the cases for Poisson and negative binomial can also be found in Ref. [7]. Figure 1 shows the typical multiplicity distributions as an example for proton, anti-proton, and net-proton for two different $\sqrt{s_{N N}}=7.7$ and $200 \mathrm{GeV}$ by taking their corresponding mean values measured by STAR experiment [9].

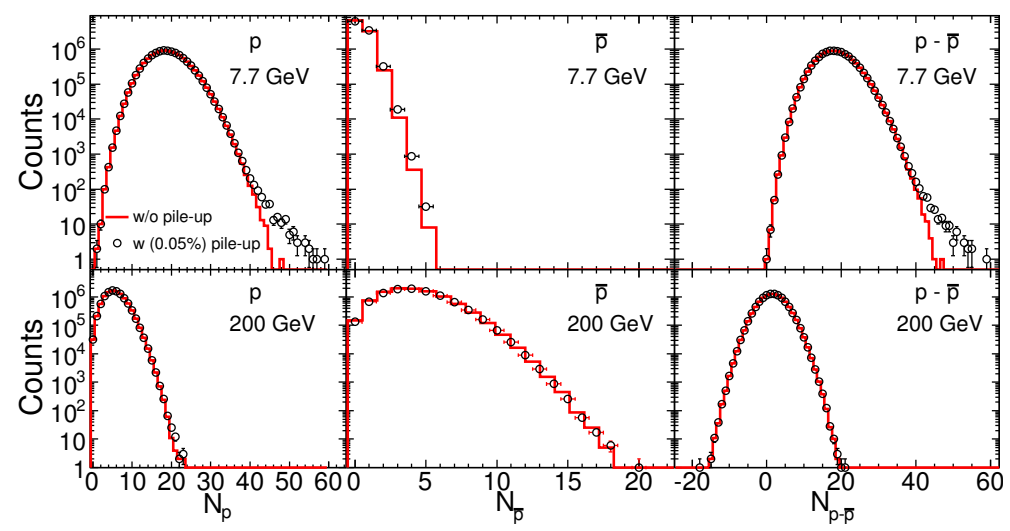

Figure 1: (Color online) The proton, anti-proton and net-proton multiplicity distributions are shown with (open symbol) and without (solid line) event pile-up for $\sqrt{s_{N N}}=7.7 \mathrm{GeV}$ and $200 \mathrm{GeV}$. The proton and antiproton multiplicities from minimum bias events as pile-up are added with the individual $\mathrm{p}$ and $\bar{p}$ distributions assuming each of the distribution as NBD.

In Fig. 1, the $p$ and $\bar{p}$ multiplicities from the central events are combined with $0.05 \%$ (5 
pile-up events in $10^{4}$ events) of the randomly selected $p$ and $\bar{p}$ multiplicities from minimum bias events. Some fraction of excess protons due to pile-up can clearly be seen as compared to a purely NBD distribution at $\sqrt{s_{N N}}=7.7 \mathrm{GeV}$. These excess events also reflect in the $N_{\text {diff }}$ distributions. Events with higher $p$ or $\bar{p}$ multiplicities will have larger pile-up effects, which can be observed in the proton multiplicity distribution. The effect of event pile-up is more visible in the proton distribution at $\sqrt{s_{N N}}=7.7 \mathrm{GeV}$ as compared to $200 \mathrm{GeV}$. Since at lower energies the mean number of protons is larger as compared to higher energies, therefore, the effect of mixing a central event with another central (or minimum bias) event, is more pronounced. However, in experimentally measured $p$ and $\bar{p}$ multiplicity distributions, it is difficult to figure out these events, as the excess is very small and it may look like a real event multiplicity distribution.

The binomial distributions are constructed using the mean $\left(C_{1}\right)$ and variance $\left(C_{2}\right)$ values of the proton and anti-proton multiplicity distributions. The multiplicity distributions are assumed to be a binomial distribution as

$$
B(n, p)=\frac{n !}{k !(n-k) !} p^{k}(1-p)^{n-k}
$$

where $k$ is the observed multiplicity, $n$ is the particles being produced and $p$ is the probability to measure it. The $C_{1}$ and $C_{2}$ are related to the above parameters as $C_{1}=n p$ and $C_{2}=n p(1-$ $p$ ). Afterwards, the net-proton distribution is constructed using these two binomial distributions. Figures 2 and 3 show the cumulant ratios as a function of $\sqrt{s_{N N}}$ for different fraction of pile-up events from central and minimum bias collisions, respectively mixed with the $p$ and $\bar{p}$ multiplicity distributions from the central collisions.
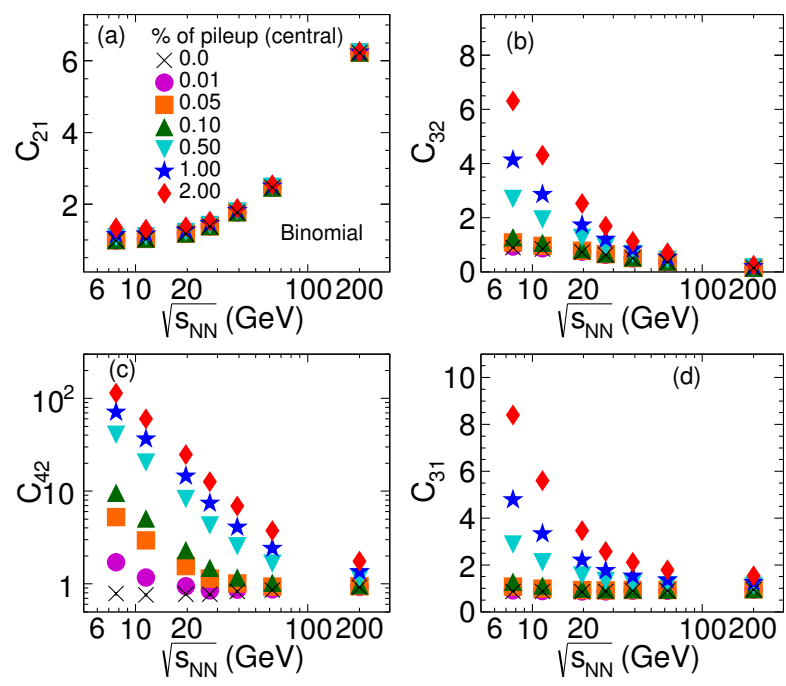

Figure 2: (Color online) Variation of cumulant ratios (C2/C1, C3/C2, C4 /C2 , and C3 /C1 ) of net-proton distributions as a function of $\sqrt{s_{N N}}$ for different fraction of pile-up events for central $(0 \%-5 \%) \mathrm{Au}+\mathrm{Au}$ collisions. The individual $\mathrm{p}$ and $\bar{p}$ multiplicity distributions are assumed to be binomial. The pile-up events from central collisions are mixed with the original distributions from the same centrality.

It is evident that the $C_{32}, C_{42}$, and $C_{31}$ ratios show strong dependence on energy and the fraction of added pile-up events. We have tested the effect of pile-up after considering the NBD and Poisson 

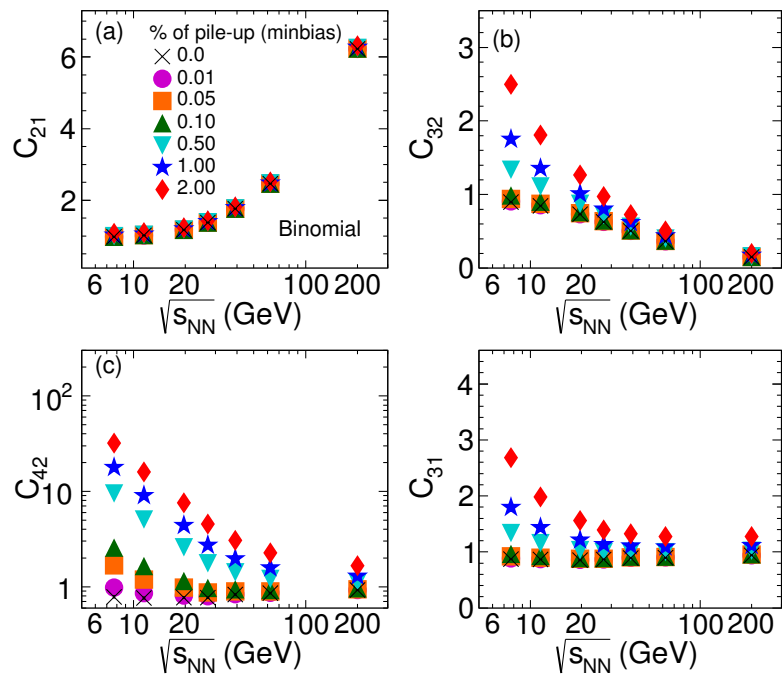

Figure 3: (Color online) The pile-up events from minimum bias collisions are mixed with the original distributions from the same centrality.

distributions as $\mathrm{p}$ and $\bar{p}$ multiplicities and the results are found to be qualitatively similar to the binomial distribution.

\section{Contribution of Stopped Proton Fluctuations}

In this section we show the influence of stopped protons $\left(p^{s t o p}\right)$ on the fluctuations of final netproton multiplicities produced in heavy-ion collisions. Experimentally measured protons $\left(p^{\text {incl }}\right)$ contain contribution from various processes such as secondaries from higher mass resonance decays, production process $\left(p^{p r o d}\right)$, and protons from the baryon stopping. Further, these contributions also fluctuate from event to event and can contaminate the dynamical fluctuations due to the critical point. We present the contribution of stopped proton and produced proton in the net-proton multiplicity fluctuations in Au+Au collisions measured by STAR experiment at RHIC at different collision energies. At each studied energy, the fraction of stopped proton yield is assumed to fluctuate according to a Gaussian distribution [8]. The produced net-proton fluctuations are estimated after subtracting the stopped proton contribution from the inclusive proton multiplicity distribution.

For this exercise, as the measured data for individual distributions is not available, we tune the measured cumulants with the binomial assumption. As a proof of principle, we calculate the individual cumulants of measured proton, antiproton and net-proton multiplicities and compare them with the experimentally measured cumulants as is shown in Fig 4.

Experimentally, it is not trivial to identify a proton originating from stopping or production, hence, the correction for the stopped protons to the net-proton multiplicity distribution can not be applied experimentally. The fraction of stopped and produced proton contributions in the inclusive proton distribution are estimated by taking the mean number of $p^{\text {incl }}$ [9] and $p^{\text {stop }}$ [10]. The stopped and produced proton fractions are calculated as: $f^{\text {stop }}=\left(C_{1}\right.$ of $p^{\text {stop }} / C_{1}$ of $\left.p^{\text {incl }}\right)$ and $f^{\text {prod }}=\left[\left(C_{1}\right.\right.$ of $p^{\text {incl }}-C_{1}$ of $\left.p^{\text {stop }}\right) / C_{1}$ of $\left.p^{\text {incl }}\right]$, respectively. The stopped proton and produced proton distribu- 


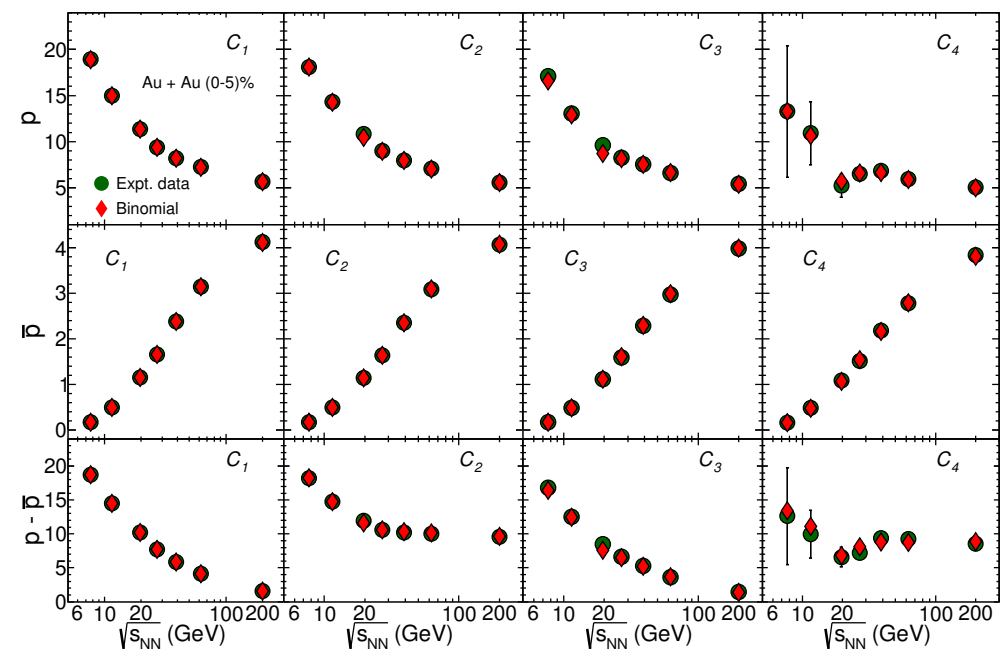

Figure 4: (Color online) The pile-up events from minimum bias collisions are mixed with the original distributions from the same centrality.

tions are constructed on event-by-event basis from the inclusive proton distribution weighted by corresponding $f^{\text {stop }}$ and $f^{\text {prod }}$ fractions, respectively. Hence, the mean of the original $p^{\text {incl }}$ distribution change to the corresponding mean values of $p^{\text {stop }}$ and $p^{\text {prod }}$, without modifying the shape of the distribution. The resulting $p^{\text {stop }}$ and $p^{\text {prod }}$ distributions also remain binomial. These factorized multiplicity distributions along with the anti-proton multiplicity distributions are further used for the study of net-proton fluctuation at various collision energies.
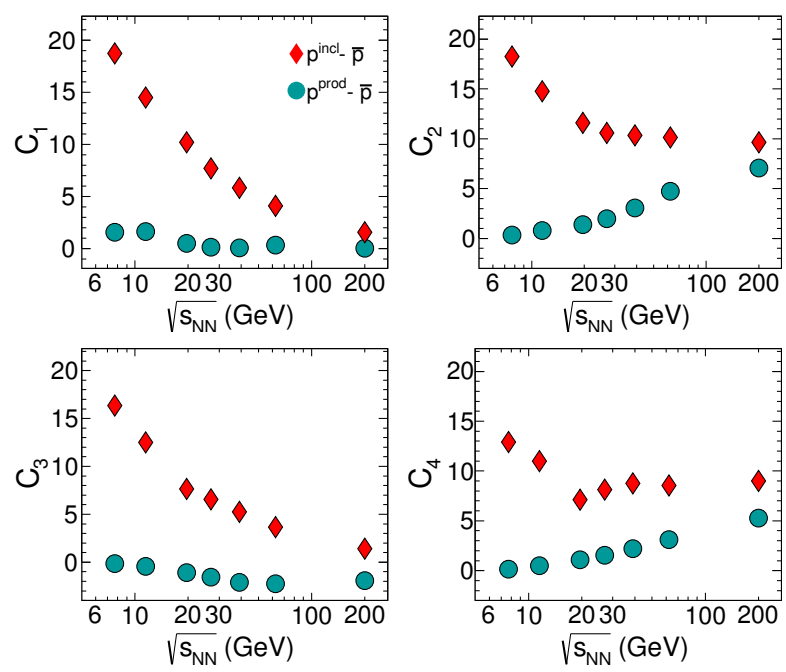

Figure 5: (Color online) Variation of cumulants of net-proton multiplicity distributions as a function of $\sqrt{s_{N N}}$ in most central (0\%-5\%) Au+Au collisions obtained from binomial assumption.

Figure 5 shows the individual cumulants $\left(C_{1}, C_{2}, C_{3}\right.$, and $\left.C_{4}\right)$ obtained from $p^{\text {incl }}-\bar{p}$ and $p^{\text {prod }}$ $\bar{p}$ distributions as a function of $\sqrt{s_{N N}}$. The cumulants of the difference distribution obtained from 
$p^{\text {incl }}-\bar{p}$ decrease as a function of collision energy. After subtracting the stopping contributions from the $p^{\text {incl }}$ distribution, the average number of produced protons and anti-protons are similar, which gives a smaller value of $C_{1}$ in $p^{\text {prod }}-\bar{p}$ distribution and it is almost independent of collision energy. The cumulants $\left(C_{1}\right.$ and $\left.C_{3}\right)$ of the difference distributions obtained from $p^{\text {prod }}-\bar{p}$ multiplicities show small energy dependence, whereas $C_{2}$ and $C_{4}$ cumulants systematically increase with $\sqrt{s_{N N}}$.

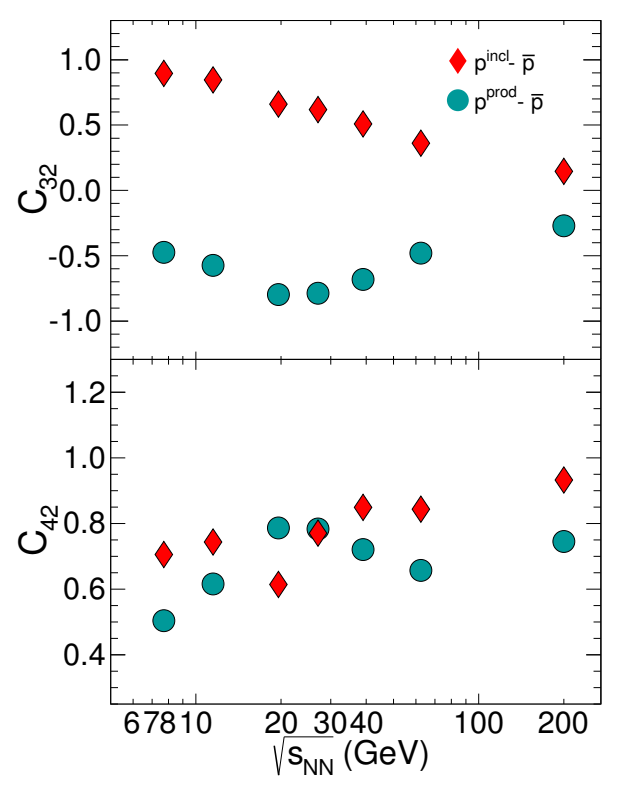

Figure 6: (Color online) Variation of cumulant ratios $C_{3} / C_{2}$ and $C_{4} / C_{2}$ of net-proton distributions as a function of $\sqrt{s_{N N}}$ in most central (0-5\%) Au+Au collisions obtained from binomial assumption.

Figure 6 shows the energy dependence of ratios of cumulants $\left(C_{3} / C_{2}\right.$ and $\left.C_{4} / C_{2}\right)$ for net-proton $p^{\text {diff }}$ distributions obtained from $p^{\text {incl }}, p^{\text {prod }}$, and $\bar{p}$ multiplicity distributions. The $C_{3} / C_{2}$ ratios of net-proton distributions by taking inclusive protons and anti-protons decrease with collision energies, similar observation is also reported by STAR experiment [5]. Further, the $C_{3} / C_{2}$ ratios from the produced protons and antiprotons as a function of $\sqrt{s_{N N}}$, are found to be minimum around $19.6 \mathrm{GeV}$. The $C_{4} / C_{2}\left(=\kappa \sigma^{2}\right)$ ratios from $p^{\text {incl }}-\bar{p}$ shows deviation from the Poisson expectation for lower collision energies and maximum deviation is observed at $19.6 \mathrm{GeV}$, which is also observed in the experimental data. While taking the inclusive proton with $\bar{p}$, a minimum is observed at $19.6 \mathrm{GeV}$ for $C_{4} / C_{2}$ ratios, whereas by taking the produced proton and antiproton a maximum is observed at the same energy. After removing the stopping contribution from the inclusive proton distribution, the $C_{3} / C_{2}$ and $C_{4} / C_{2}$ ratios of the net-proton ( $p^{\text {diff }}$ ) multiplicity distribution shows non-monotonic behavior at $\sqrt{S_{N N}}=19.6 \mathrm{GeV}$, which may indicate the presence of a critical point or phase-transition in heavy-ion collisions.

\section{Summary}

To summarize the present work, we have emphasized the importance of residual event pile-up for net-proton higher moment analysis. It is demonstrated that even a very small fraction of the 
pile-up events can change the higher cumulants significantly especially at lower center-of-mass energies. This issue is even more important for the fixed target experiments like CBM where the collision rates will be even higher. Also, we observe a significant effect in cumulants and their ratios of net-proton multiplicity distributions due to pile-up events, particularly at lower energies. It might be crucial to estimate the fraction of pile-up events in the data sample while interpreting the experimental observable for the critical point.

Further, we demonstrate a Monte-Carlo method to disentangle the contribution of the stopped protons and produced protons from the inclusive proton fluctuations in the heavy-ion collisions. The $\sqrt{s_{N N}}$ dependence of the efficiency corrected cumulants and their ratios for proton, antiproton, and net-proton multiplicities are compared with the same obtained from a binomial distributions. Whereas, after removing the stopping contribution from the inclusive proton distribution, the $C_{3} / C_{2}$ and $C_{4} / C_{2}$ ratios of the net-proton multiplicity distribution shows non-monotonic behavior around $\sqrt{s_{N N}}=19.6 \mathrm{GeV}$. This may indicate the presence of a critical point or a phase-transition in high-energy heavy-ion collisions.

\section{References}

[1] M. A. Stephanov, K. Rajagopal and E. V. Shuryak, Phys. Rev. Lett. 81, 4816 (1998).

[2] M. A. Stephanov, K. Rajagopal and E. V. Shuryak, Phys. Rev. D 60, 114028 (1999).

[3] A. Bazavov et al., Phys. Rev. Lett. 109, 192302 (2012).

[4] P. Garg, D. K. Mishra, P. K. Netrakanti, B. Mohanty, A. K. Mohanty, B. K. Singh and N. Xu, Phys. Lett. B 726, 691 (2013).

[5] L. Adamczyk et al. [STAR Collaboration], Phys. Rev. Lett. 112, 032302 (2014).

[6] A. Adare et al. [PHENIX Collaboration], Phys. Rev. C 93, 011901 (2016).

[7] P. Garg and D. K. Mishra, Phys. Rev. C 96, 044908 (2017).

[8] D. K. Mishra and P. Garg, arXiv:1706.04012 [nucl-th].

[9] Cumulants of proton and anti-proton distribution measured by STAR experiment.

[10] D. Thakur, S. Jakhar, P. Garg and R. Sahoo, Phys. Rev. C 95, 044903 (2017). 